\title{
The legacy of the OPERA experiment on neutrino oscillations
}

\section{Giuliana Galati ${ }^{* \dagger}$}

Università degli Studi di Napoli "Federico II" and INFN

E-mail: giuliana.galati@na.infn.it

\begin{abstract}
The OPERA experiment has conclusively observed the appearance of tau neutrinos in the muon neutrino CNGS beam. High purity samples of $v_{e}, v_{\mu}$ and $v_{\tau}$ charged current neutrino interactions, as well as neutral current interactions were isolated. Recent results obtained using the full dataset to test the three-flavor neutrino oscillation model are discussed. Constraints on the existence of a light sterile neutrino, derived using for the first time tau and electron neutrino appearance channels, are also presented. A significant fraction of the sterile neutrino parameter space allowed by LSND and MiniBooNE experiments is excluded at 90\% C.L. In particular, the best-fit oscillation parameter values obtained by MiniBooNE are excluded at $3.3 \sigma$ significance.
\end{abstract}

European Physical Society Conference on High Energy Physics - EPS-HEP2019 -

10-17 July, 2019

Ghent, Belgium

\footnotetext{
* Speaker.

${ }^{\dagger}$ On behalf of the OPERA Collaboration
} 


\section{Introduction}

The OPERA experiment [1] was designed to conclusively prove the existence of $v_{\mu} \rightarrow v_{\tau}$ oscillations. The direct appearance search was based on the detection of $\tau$ leptons produced in $v_{\tau}$ charged current (CC) interactions. The challenge in detecting the short-lived $\tau$ lepton (c $\tau=$ $87 \mu \mathrm{m}$ ), produced in the $\mathrm{CC} v_{\tau}$ interactions, out of almost twenty thousands $v_{\mu}$ interactions, was achieved using the nuclear emulsion technique that features micrometric spatial resolution. Moreover, thanks to the very high granularity of the emulsion films, showers induced by electrons could be distinguished from those induced by neutral pions, thus allowing the detection of charged current interactions of electron neutrinos.

\section{The CNGS beam and the OPERA detector}

The OPERA detector was located in the underground Gran Sasso Laboratory (LNGS), $730 \mathrm{~km}$ away from the neutrino source, in the high energy long-baseline CERN to LNGS beam (CNGS) $[2,3]$. The average neutrino energy was $\sim 17 \mathrm{GeV}$, the $\bar{v}_{\mu}$ contamination amounted to $2.1 \%$ in terms of interactions, the $v_{e}$ and $\bar{v}_{e}$ together were below $1 \%$, and the number of prompt $v_{\tau}$ was negligible. The detector was an hybrid apparatus consisting of an emulsion/lead target complemented by electronic detectors (see Fig. 2 a)). It was made up of two identical super-modules aligned along the CNGS beam direction, each made of a target section and a muon spectrometer. Each target section consisted of a multi-layer array of 31 target walls interleaved with pairs of planes of plastic scintillator strips. Target walls were made of 150000 Emulsion Cloud Chamber target units, called bricks (see Fig. 2 b)). Each brick consisted of 57 emulsion films, $300 \mu \mathrm{m}$ thick, interleaved with 56 lead plates, $1 \mathrm{~mm}$ thick, for a total mass of $8.3 \mathrm{~kg}$. The electronic detectors were used to identify the brick containing the neutrino interaction, for muon identification and the determination of its charge and momentum.
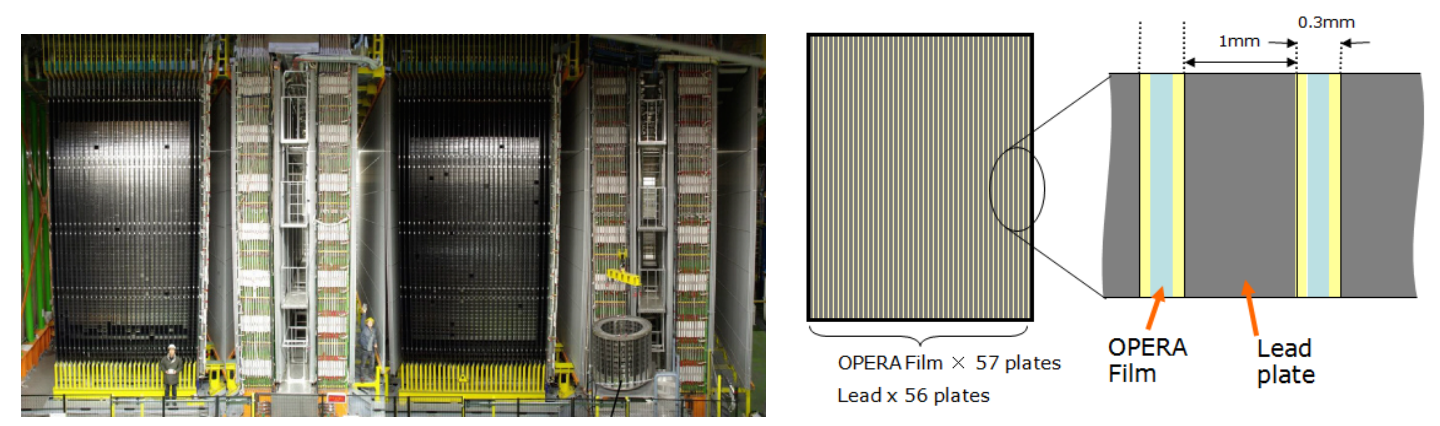

Figure 1: a) On the left a photo of the OPERA detector; b) on the right a scheme of the OPERA brick

\section{Event selection and analysis}

Every brick containing an interaction was extracted from the target wall to allow for emulsion film development, scanning and for the search for $\tau$ decays, carried out in the emulsion scanning laboratories in Europe and Japan with automated optical microscopes. If a secondary vertex 
was found, a full kinematic analysis was performed combining the measurements in the nuclear emulsion with data from the electronic detectors. The momentum of charged particles could be measured in emulsions by Multiple Coulomb Scattering [4].

Detected electromagnetic showers were carefully inspected, by visual scan, to assess whether produced by a single particle. Thanks to the high granularity of the OPERA nuclear emulsions, it was possible to identify an e-pair from $\gamma$ conversion when the e-pair tracks were separated by more than $1 \mu \mathrm{m}$. Once the origin of the e.m. shower was confirmed as due to a single charged particle, the event was classified as $v_{e}$ candidate.

For muons crossing the spectrometers, the momentum was measured with a resolution better than $22 \%$ up to $30 \mathrm{GeV} / \mathrm{c}$, and the charge determined [5].

\section{4. $v_{\mu} \rightarrow v_{\tau}$ oscillations}

Runs with CNGS neutrinos were successfully carried out from 2008 to 2012, for a total intensity of $17.97 \cdot 10^{19}$ protons on target (p.o.t.). In 2015, the observation of five $v_{\tau}$ candidates $[6,7,8,9,10]$, with a background of $0.25 \pm 0.05$ events, allowed to exclude the absence of $v_{\mu} \rightarrow v_{\tau}$ oscillations with a $5.1 \sigma$ significance, as discussed in the paper [10].

By exploiting the unique capability of the OPERA experiment to identify all three neutrino flavours, constrains on the oscillation parameters were set by a joint oscillation fit of all datasets. New analysis strategy was developed, fully exploiting the features expected for $v_{\tau}$ events. A multivariate approach to improve signal to noise separation was applied to candidate events selected by means of moderately tight topological and kinematical cuts. Different multivariate techniques were considered and their performances for signal to background discrimination compared. The one with the best discrimination power was the Boosted Decision Tree (BDT).

Details about the new selection method are reported in [11]. The total expected signal, after the application of selection criteria, amounts to $(6.8 \pm 1.4)$ events, whereas the total background expectation is $(2.0 \pm 0.4)$ events. Ten events were observed [11].

The statistical analysis implemented to re-evaluate the significance for the $v_{\tau}$ appearance was based on an extended likelihood constructed as the product of a probability density function given by the BDT response, a Poisson probability term which takes into account the number of observed events and the expected background in each decay channel, and a Gaussian term which accounts for systematics. The null hypothesis was excluded with the improved significance of $6.1 \sigma$ [11].

\subsection{First measurement of $\left|\Delta m_{23}^{2}\right|$ in appearance mode and of $v_{\tau} \mathbf{C C}$ cross-section on Lead}

The number of observed $v_{\tau}$ candidates after background subtraction is a function of the product of $v_{\tau}$ CC cross-section $\left(\sigma_{v_{\tau}}^{C C}\right)$ and the oscillation parameter $\left|\Delta m_{23}^{2}\right| .\left|\Delta m_{23}^{2}\right|$ was evaluated for the first time in appearance mode: assuming $\sin ^{2} 2 \theta_{23}=1,\left|\Delta m_{32}^{2}\right|$ is equal to $\left(2.7_{-0.6}^{+0.7}\right) \cdot 10^{-3} \mathrm{eV}^{2}$. The result is consistent within $1 \sigma$ with the measurements performed in disappearance mode by other experiments and with the Particle Data Group best fit [12].

Tau-neutrino CC cross-section on the lead target was estimated equal to $\left(5.1_{-2.0}^{+2.4}\right) \cdot 10^{-36} \mathrm{~cm}^{2}$, with $\left|\Delta m_{32}^{2}\right|=2.50 \cdot 10^{-3} \mathrm{eV}^{2}$, as shown in Fig. [?]. This result is the first measurement of the $v_{\tau} \mathrm{CC}$ cross-section with a negligible contamination from $\bar{v}_{\tau}$. 


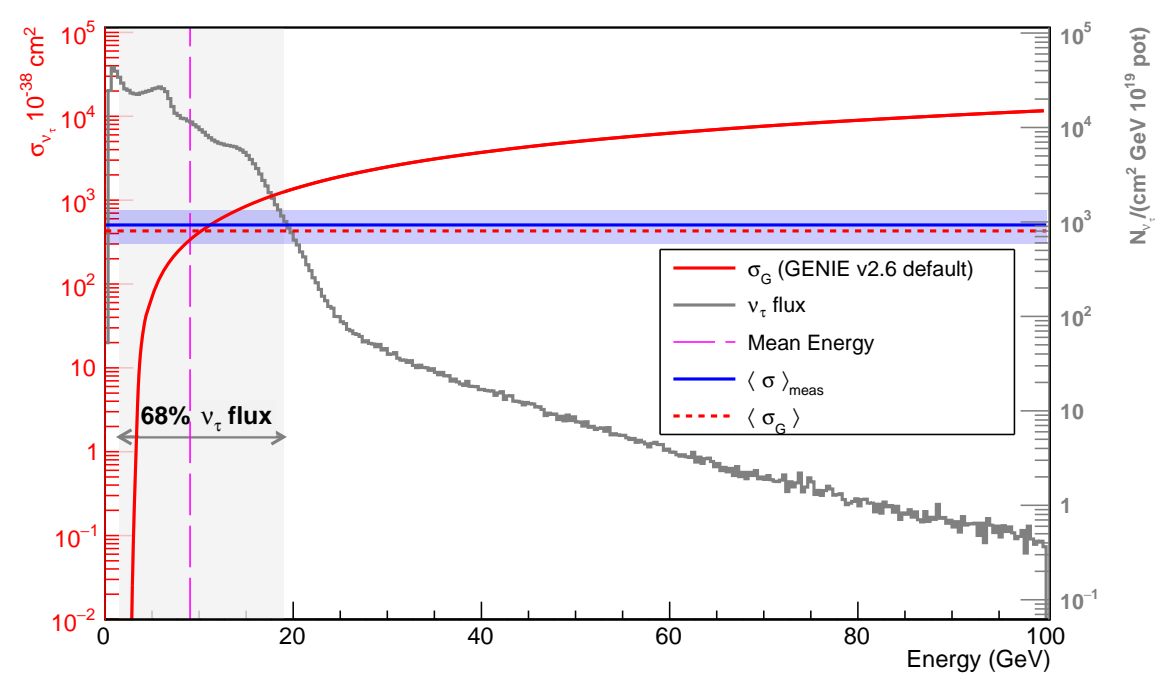

Figure 2: Flux-averaged measurement of the $\mathrm{CC} \sigma_{v_{\tau}}$ on a lead target. The horizontal band includes the central $68 \%$ of the flux.

\section{$4.2 v_{\tau}$ lepton number}

The lepton number of $v_{\tau}$ had never been observed. In the muonic channel, the OPERA experiment could distinguish neutrinos from anti-neutrinos looking at the charge of the muon produced in $\tau$ decays, which was measured to be negative at $5.6 \sigma$ level for the $\tau \rightarrow \mu$ candidate. Performing a dedicated BDT analysis which included also the background due to the $2 \% \bar{v}_{\mu}$ beam contamination, we obtained the first direct evidence for the leptonic number of $\tau$ neutrinos with a significance of $3.7 \sigma[11]$.

\section{5. $v_{\mu} \rightarrow v_{e}$ oscillations}

The tracking capabilities of emulsions allow to identify electrons produced in CC interactions of $v_{e}$, and therefore to search for $v_{e}$ appearance from $v_{\mu} \rightarrow v_{e}$ oscillations. Thirtyfive $v_{e}$ candidate events were observed [13], compatible with the expected $v_{e}$ events from the beam contamination $\left(30.7 \pm 0.9\right.$ (stat.) \pm 3.1 (syst.)), and two main sources of background: $\pi^{0}$ misidentified as an electron $0 \mu$ events and $v_{\tau} \mathrm{CC}$ interactions with lepton decaying into an electron, amounting to $1.2 \pm 0.5$ (stat.) \pm 0.2 (syst.) events.

The number of observed events is compatible with what expected in the 3-flavour oscillation model. Indeed, assuming: $\sin ^{2}\left(2 \theta_{13}\right)=0.098, \sin ^{2} 2 \theta_{23}=1, \Delta m_{32}^{2}=\Delta m_{31}^{2}=2.44 \times 10^{3} \mathrm{eV}^{2}, \delta_{C P}=$ 0 , and neglecting matter effects, $N_{\text {exp }}^{3 v}=34.3 \pm 1.0$ (stat.) \pm 3.4 (syst.) $v_{e} \mathrm{CC}$ events are expected to be detected in the whole energy range, including background events.

\section{Search for sterile neutrinos via $v_{\mu} \rightarrow v_{\tau}$ and $v_{\mu} \rightarrow v_{e}$ oscillations}

The $v_{\tau}$ and $v_{e}$ appearance channels were combined for the first time to constrain oscillation parameters of the so-called $3+1$ neutrino mixing model [14]. Observed neutrino oscillation anoma- 
lies, if interpreted in terms of one additional sterile neutrino, suggest $\left|\Delta m_{41}^{2}\right|$ values at the $\mathrm{eV}^{2}$ scale. In the framework of the $3+1$ model, for $\Delta m_{41}^{2}>0.1 \mathrm{eV}^{2}$, the measured $90 \%$ C.L. upper limit on the mixing term $\sin ^{2} 2 \theta_{\mu \tau}=4\left|U_{\mu 4}\right|^{2}\left|U_{\tau 4}\right|^{2}$ is 0.10 (see Fig. 6 a), while on $\sin ^{2} 2 \theta_{\mu e}=4\left|U_{e 4}\right|^{2}\left|U_{\mu 4}\right|^{2}$ is 0.019 (see Fig. 6 b)), independently of the mass hierarchy of the three standard neutrinos. Therefore, the MiniBooNE best-fit [15] values $\left(\Delta m_{41}^{2}=0.041 \mathrm{eV}^{2}, \sin ^{2} 2 \theta_{\mu e}=0.92\right)$ are excluded with $3.3 \sigma$ significance.

Appearance channels have been jointly used also to obtain constraints on three-flavor model parameters, obtaining $\theta_{23}=0.78_{-0.31}^{+0.32} \mathrm{rad}(1 \sigma$ C.L. $)$ and $0 \leq \theta_{13} \leq 0.20 \mathrm{rad}(1 \sigma$ C.L. $)$.
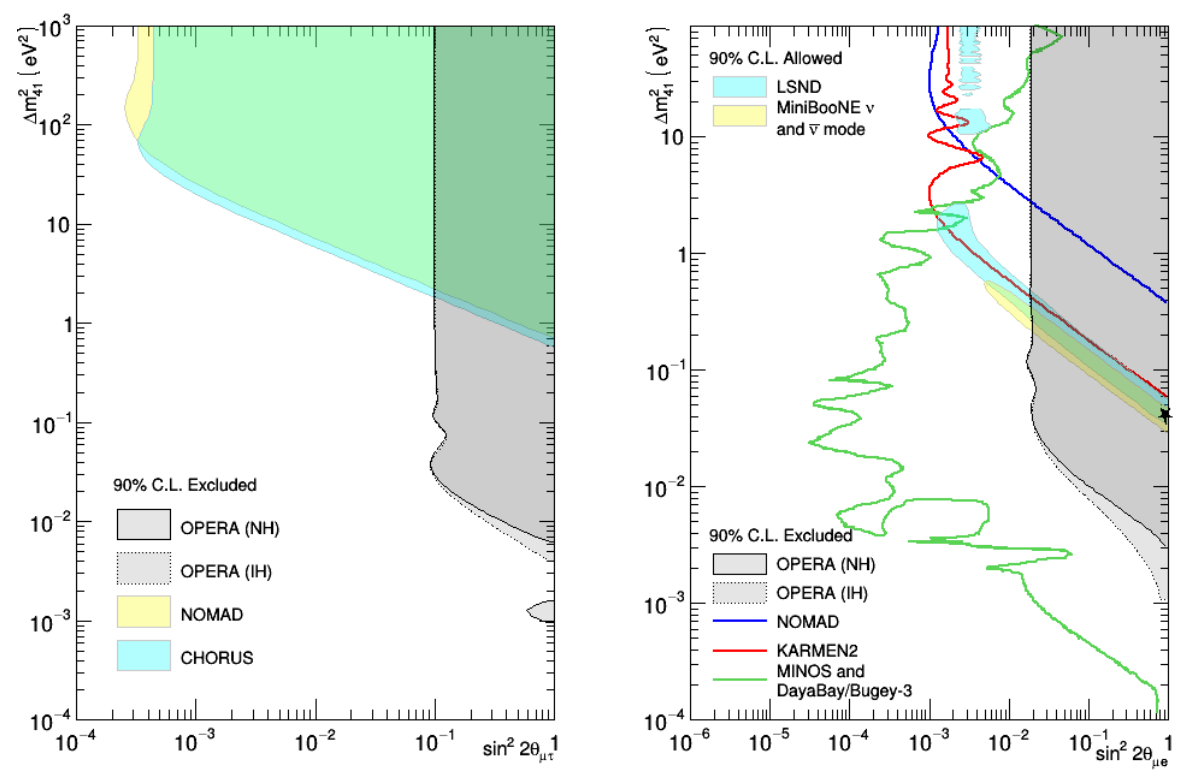

Figure 3: a) On the left, OPERA $90 \%$ CL exclusion region in the $\Delta m_{41}^{2}$ and $\sin ^{2} 2 \theta_{\mu \tau}$ parameter space for the normal (NH, solid line) and inverted (IH, dashed line) hierarchy of the three standard neutrino masses. The exclusion regions by NOMAD [16] and CHORUS [17] are also shown; b) on the right, OPERA 90\% CL exclusion region in the $\Delta m_{41}^{2}$ and $\sin ^{2} 2 \theta_{\mu \tau}$ parameter space for the normal (NH, solid line) and inverted (IH, dashed line) hierarchy of the three standard neutrino masses. The exclusion regions by NOMAD [16] and CHORUS [17] are also shown.

\section{7. $v_{\mu}$ disappearance analysis}

The $v_{\mu} \rightarrow v_{\mu}$ disappearance channel was explored using a dedicated sample of OPERA electronic detector data. The disappearance oscillation probability was estimated by the ratio NClike/CC-like event. The uncertainty on the $v_{\mu}$ flux was estimated to be $15 \%$. Being experimentally not possible to reconstruct the energy of an incoming neutrino in NC events, NC-like/CC-like event rate was evaluated as a function of the measured energy deposited in the target. Assuming all other mixing parameters equal to the global fit central values [18], an upper limit $\left|\Delta m_{32}^{2}\right| \leq 4.1 \times 10 \mathrm{eV}^{2}$ at $90 \%$ C.L. was obtained. 


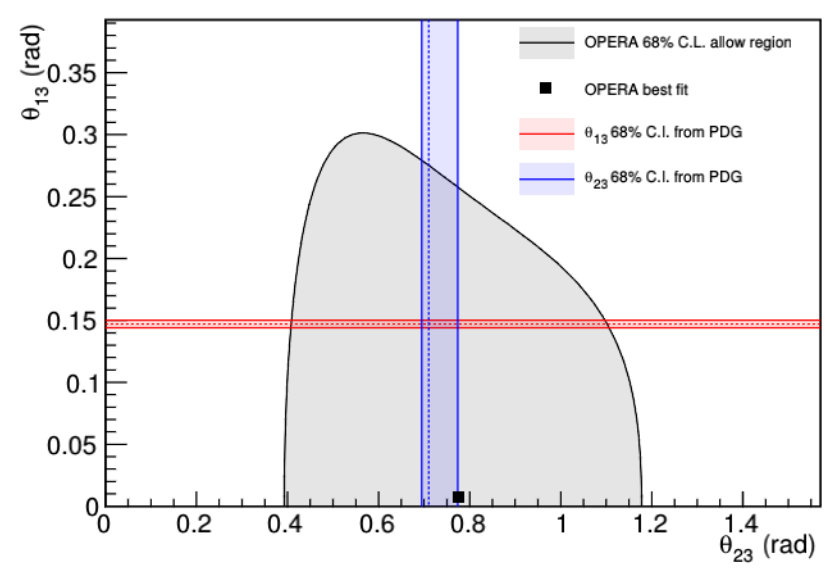

Figure 4: OPERA 68\% C.L. allowed region in the $\theta_{13}$ and $\theta_{23}$ parameter space for the normal hierarchy of the three standard neutrino masses. Red and blue dashed lines (areas) represent $1 \sigma$ confidence interval obtained from the global best fit values (Table 14.1 in [?], $3 \sigma$ allowed ranges) for $\theta_{13}$ and $\theta_{23}$ respectively.

\section{Conclusions}

The OPERA experiment took data from 2008 to 2012. Ten $v_{\tau}$ candidates were observed and the non-null observation of $v_{\mu} \rightarrow v_{\tau}$ oscillations was excluded at $6.1 \sigma$ [19]. Furthermore, the unique feature of the OPERA experiment to identify all 3 neutrino flavours was exploited in order to put constraints on the oscillation parameters by a joint oscillation fit of all datasets.

\section{References}

[1] M. Güler et al., An appearance experiment to search for $v_{\mu} \rightarrow v_{\tau}$ oscillations in the CNGS beam: experimental proposal, Tech. Rep. CERN-SPSC-2000-028. LNGS-2000-25. SPSC-P-318, CERN, Geneva, Jul, 2000.

[2] R. Acquafredda et al., The OPERA experiment in the CERN to Gran Sasso neutrino beam, JINST 4 (2009) P04018.

[3] OPERA collaboration, R. Acquafredda et al., First events from the CNGS neutrino beam detected in the OPERA experiment, New J. Phys. 8 (2006) 303, [hep-ex/ 0611023 ].

[4] OPERA collaboration, N. Agafonova et al., Momentum measurement by the Multiple Coulomb Scattering method in the OPERA lead emulsion target, New J. Phys. 14 (2012) 013026, [1106.6211].

[5] OPERA collaboration, N. Agafonova et al., Study of neutrino interactions with the electronic detectors of the OPERA experiment, New J. Phys. 13 (2011) 053051, [1102 . 1882].

[6] OPERA COllaboration collaboration, N. Agafonova et al., Observation of a first $v_{\tau}$ candidate in the OPERA experiment in the CNGS beam, Phys.Lett. B691 (2010) 138-145, [1006.1623].

[7] OPERA COLlaboration collaboration, N. Agafonova et al., New results on $v_{\mu} \rightarrow v_{\tau}$ appearance with the OPERA experiment in the CNGS beam, JHEP 11 (2013) 036, [1308 .2553]. 
[8] OPERA COLlaboration collaboration, N. Agafonova et al., Evidence for $v_{\mu} \rightarrow v_{\tau}$ appearance in the CNGS neutrino beam with the OPERA experiment, Phys. Rev. D89 (2014) 051102, [1401.2079].

[9] OPERA COLlaboration collaboration, N. Agafonova et al., Observation of tau neutrino appearance in the CNGS beam with the OPERA experiment, PTEP 2014 (2014) 101C01, [1407.3513].

[10] OPERA Collaboration collaboration, N. Agafonova et al., Discovery of $\tau$ Neutrino Appearance in the CNGS Neutrino Beam with the OPERA Experiment, Phys. Rev. Lett. 115 (2015) 121802, [1507.01417].

[11] OPERA collaboration, N. Agafonova et al., Final results of the OPERA experiment on $v_{\tau}$ appearance in the CNGS beam, Phys. Rev. Lett. 120 (2018) 211801, [1804. 04912 ].

[12] Particle Data Group collaboration, C. Patrignani et al., Review of Particle Physics, Chin. Phys. C40 (2016) 100001.

[13] OPERA collaboration, N. Agafonova et al., Final results of the search for $v_{\mu} \rightarrow v_{e}$ oscillations with the OPERA detector in the CNGS beam, JHEP 06 (2018) 151, [1803.11400].

[14] OPERA collaboration, N. Agafonova et al., Final results on neutrino oscillation parameters from the OPERA experiment in the CNGS beam, Phys. Rev. D100 (2019) 051301, [1904.05686].

[15] MiniBoone Collaboration collaboration, A. A. Aguilar-Arevalo, B. C. Brown, L. Bugel, G. Cheng, J. M. Conrad, R. L. Cooper et al., Significant excess of electronlike events in the miniboone short-baseline neutrino experiment, Phys. Rev. Lett. 121 (Nov, 2018) 221801.

[16] NOMAD collaboration, P. Astier et al., Final NOMAD results on muon-neutrino $\rightarrow$ tau-neutrino and electron-neutrino $\rightarrow$ tau-neutrino oscillations including a new search for tau-neutrino appearance using hadronic tau decays, Nucl. Phys. B611 (2001) 3-39, [hep-ex/ 0106102 ].

[17] CHORUS collaboration, E. Eskut et al., Final results on $n u(m u) \rightarrow n u($ tau $)$ oscillation from the CHORUS experiment, Nucl. Phys. B793 (2008) 326-343, [0710 . 3361].

[18] Particle Data Group collaboration, M. Tanabashi et al., Review of Particle Physics, Phys. Rev. D98 (2018) 030001.

[19] OPERA collaboration, N. Agafonova et al., Discovery of $\tau$ Neutrino Appearance in the CNGS Neutrino Beam with the OPERA Experiment, Phys. Rev. Lett. 115 (2015) 121802, [15 07.01417$].$ 